\title{
Crowdsourcing with Trembles: Incentive Mechanisms for Mobile Phones with Uncertain Sensing Time
}

\author{
Shiyu $\mathrm{Ji}^{\dagger}$, Tingting Chen*, Fan $\mathrm{Wu}^{\S}$ \\ $\dagger$ Oklahoma State University, shiyu@cs.okstate.edu $\S$ Shanghai Jiao Tong University, fwu@cs.sjtu.edu.cn \\ * California State Polytechnic University, Pomona, tingtingchen@csupomona.edu
}

\begin{abstract}
Mobile phone sensing has become increasingly popular since it can collect and analyze real-time data anywhere anytime, especially with the help of mobile phone users via crowdsourcing. In order to stabilize the mobile crowdsourcing at a massive scale, incentive mechanisms are needed not only to stimulate the users of mobile phones to participate in sensing, but also to incentivize the organizer of the sensing tasks with maximum service time and payoff. In this paper, we study a practical problem in mobile sensing, i.e., sensing time uncertainty, which may lead to failures of existing incentive mechanisms. In particular, we model this problem as a perturbed Stackelberg game in which mobile phone users may actually conduct sensing tasks during different periods of time rather than what they intend, like through a trembling hand. We find that there exist Trembling-Hand Perfect Equilibria (THP) given proper rewards. After characterizing THPs in this game with rigorous analysis, we design incentive mechanisms that can achieve a THP with the maximum system wide total sensing time and maximum platform utility. We finally verify the correctness and efficiency of our proposed incentive mechanisms and algorithms through extensive experiments.
\end{abstract}

\section{INTRODUCTION}

Ubiquitous smarthphones with sensing capabilities have revolutionized the way people collect and analyze information. The embedded sensors such as camera, GPS, and acceleration sensors can provide first-hand surrounding information in various forms in real-time [1]. This new paradigm of pervasive data collection can boost the evolution of numerous applications [2], [3], [4], [5], [6], such as medical research [5] and the new targeted advertising in social networks [6]. Mobile phone sensing can make even wider impact, when the scale of sensing is greatly enlarged by crowdsourcing the sensing tasks to a large group of people.

A major challenge in achieving a stable crowdsourcing system for mobile phone sensing tasks is the incentive issue. The mobile sensor owners need to be incentivized to contribute to a sensing task, while the network platform (serving as the sensing task initiator and organizer) also need properly designed mechanisms to obtain the balance between good

This work was supported in part by the State Key Development Program for Basic Research of China (973 project 2012CB316201), in part by China NSF grant 61422208 and 61272443 , in part by CCF-Intel Young Faculty Researcher Program and CCF-Tencent Open Fund, in part by the Scientific Research Foundation for the Returned Overseas Chinese Scholars, State Education Ministry, and in part by Jiangsu Future Network Research Project No. BY2013095-1-10. sensing performance and low cost. Yang et al. [7] designed the first two incentive mechanisms for crowdsourcing to smartphones. Both of the mechanisms require the platform to pay the participating mobile phone users for their contributions, and guarantee desirable equilibria in the crowdsourcing process modeled either as a platform-centric game or as an user-centric auction. With the increasing scale of users, there can be multiple platforms who are connected together via networks, forming a cloud-based system. For example, Sheng et al. [8] proposed the concept of Sensing as a Service for cloud-based platforms and designed incentive mechanisms for different scenarios.

However, to the best of our knowledge, no existing research work so far has considered a critical issue in mobile crowdsourcing, i.e., the sensing time uncertainty. When the sensors are interacting with the physical world, such as collecting images or location information, data is often associated with uncertainty due to reasons like sampling errors, inaccurate measurements, etc. There are extensive crowdsensing applications leveraging data mining and learning techniques on client terminals (i.e., smartphones) [9] [10] [11]. In order to guarantee the successive data processing results, the sensing behaviors of the client terminals can be significantly influenced (e.g., repeating or extending sensing time), by some factors such as data quality [12], data source diversity [13] and interaction with big data [14]. In this way, the uncertainty in data can further propagate to the uncertainty in sensing behaviors. Although in the field of database, much research has been focused on managing data uncertainty (e.g., [12] [15]), it is not clear how sensing uncertainty affects the crowdsourcing procedure and results. Moreover, how sensing time uncertainty can impact the incentive mechanisms therein is also unknown. There are also some significant mobile phone based sensing time constraints, including the required initialization time of the sensing application [16], the maximum sensing time allowed by the battery charge [17], the reaction delay of the sensor [9], etc. These constraints can also introduce sensing time uncertainty.

With the consideration of sensing time uncertainty, incentive mechanisms for crowdsensing need to be revisited. In fact, in the presence of uncertainty, it is not clear how the users need to adjust their strategy depending on the uncertainty estimation for their own devices. For instance, if the sensing 
time uncertainty can bring significant perturbations to the sensing duration, the user may refuse to offer the very short sensing services. The reason is that he may end up with deficit by unintentionally sensing much longer than planned, and the cost of sensing is much higher than the amount of money actually paid based on the plan. Then the problem of how to stimulate the users and ensure the system performance in the presence of sensing time uncertainty becomes a realistic and challenging problem.

\section{Our contributions are as follows:}

- We are the first to investigate the impact of sensing time uncertainty in the problem of crowdsourcing to mobile phones. We model the crowdsourcing problem with sensing time uncertainty as a perturbed game, and find the expected best response of each user given others' strategies, and prove that the strategy profile consisting of the expected best responses is a Trembling-Hand Perfect Equilibrium.

- We find that in the perturbed crowdsourcing game, there will be no THP if the total reward is too small, and show that our incentive mechanism guarantees THP given sufficiently large total reward.

- We design the sensing time determination algorithm for each user to calculate its strategy in THP, which guarantees system-wide total sensing time. Our algorithm achieves linear complexity on the number of users.

- We conduct extensive experiments on our proposed incentive mechanisms. It verifies that our proposed incentive mechanism is incentive-compatible, optimal and efficient.

We organize the rest of this paper as follows. In Section II, we expatiate our proposed system models and the general assumptions. In Section III, we propose the user-aspect algorithm to calculate its own strategy. We demonstrate the simulation results in Section IV. Finally we talk about the related works in Section V and conclude this paper in Section VI. Due to space limitation, please see [18] for the proofs of the lemmas and theorems.

\section{SySTEM Model}

In this section, we will introduce the general mobile phone sensing system where our incentive mechanism is applied. We will also model the crowdsourcing game with uncertainty perturbations.

\section{A. General Mobile Phone Sensing System}

Fig. 1 shows the architecture of the general mobile phone sensing system. The system consists of a platform and numerous smartphone users. The platform may have some sensing tasks that need to be crowdsourced to smartphone users to leverage their distributed sensing resources. When planning the sensing tasks, the platform strategizes a proper reward based on its knowledge about each user, and publicizes the details of the sensing tasks. Usually, there are more than one users who will participate in the sensing tasks, and the participation of each user will yield a cost (we will expatiate it later). In order to compensate the cost, the platform gives a payment to

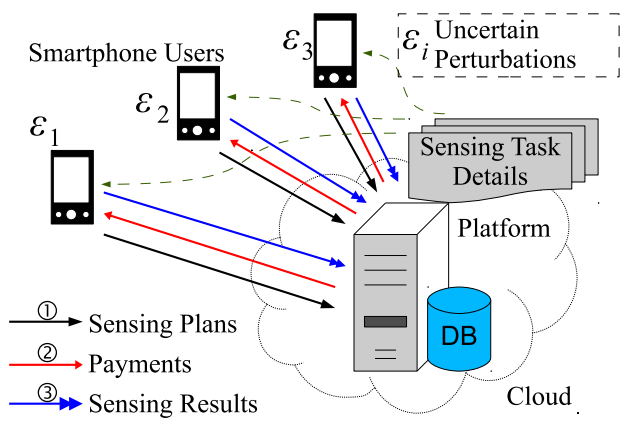

Fig. 1. General mobile phone sensing system

each user. Each participating user lets the platform know its planned sensing time. After collecting the sensing plans from all the users, the platform computes the payment for each user and sends the payments to the users instantly. The users who receive payments will execute the sensing tasks and send the sensed results to the platform.

We assume each user and the platform are rational and selfish. The users aim to earn as much reward as possible considering their own competitions. For the platform, there are two objectives: 1) to ensure the system can converge to a desirable stable state; 2) to have the sensing tasks completed with good quality and reasonably low payment.

Corresponding to the procedures described above and considering the phenomenon of sensing time uncertainty in the extensive existing crowdsourcing techniques, we provide a new model in this paper to approach the problem. Our incentive mechanism should ensure sufficient incentive for each member in our sensing system.

\section{B. Crowdsourcing Game with Uncertainty Perturbations}

We model the crowdsourced mobile sensing problem as a perturbed Stackelberg game [19], in which the platform is the leader and the users are the followers. There are two phases in the game. The platform publicizes the reward $R$ and sensing task descriptions in the first phase. The amount of the reward $R$ from the platform is a positive monetary incentive given by the platform. For the second phase, each user strategizes its own intended sensing time (strategy $t_{i}$ ) to achieve maximized utility, based on the reward and other users' conditions (including costs and perturbations). Thus, the actual sensing time of user $i$ is $\bar{t}_{i}=t_{i}+\varepsilon_{i}$, where $\varepsilon_{i}$ is the uncertainty perturbation, a bounded random variable. The perturbed game indicates that no user can predict its actual sensing time exactly. In game theory, we have a concept, the Totally Mixed Strategy Profile, to describe the scenario here.

Definition 1. (Totally Mixed Strategy Profile) A strategy profile $t=\left\{t_{i}\right\}_{i=1}^{n}$ is totally mixed if any action of any user owns a positive probability ${ }^{2}$.

\footnotetext{
${ }^{2}$ In this paper, $n$ denotes the number of all the users, and we do not distinguish the terms strategy and action.
} 
We assume each perturbation $\varepsilon_{i}$ is distributed within its bounded support interval, and the middle point of the interval is the mean of the perturbation, since the results in [20] and [21]. The support interval $I$ of the perturbation $\varepsilon_{i}$ should guarantee the actual sensing time is positive. That is, if the strategized sensing time is $t_{i}$, its support interval should be $\left(\max \left\{0, t_{i}-A_{i}\right\}, t_{i}+A_{i}\right)$, in which $A_{i}$ denotes the maximum amplitude of $\varepsilon_{i}$, and its mean is the middle point of the interval.

We denote by $\kappa_{i}$ user $i$ 's cost unit, the cost per time unit. Provided the actual sensing time $\bar{t}_{i}=t_{i}+\varepsilon_{i}$ of user $i$, its utility $\bar{u}_{i}$ is the payment $p_{i}$ distributed by the platform minus the sensing cost $\kappa_{i} \bar{t}_{i}$.

$$
\bar{u}_{i}=p_{i}-\kappa_{i} \bar{t}_{i}, \forall i \in \mathcal{U},
$$

where $\mathcal{U}$ denotes the set of all the users. We let the platform distribute the reward $R$ proportionally to each user's expected sensing time. That is $p_{i}=\frac{\mathrm{E}\left(\bar{t}_{i}\right)}{\sum_{j \in \mathcal{U}} \mathrm{E}\left(\bar{t}_{j}\right)} R$. Then the expected utility of user $i$ is:

$$
\mathrm{E}\left(\bar{u}_{i}\right)= \begin{cases}\frac{t_{i}}{\Omega} R-\kappa_{i} t_{i} & t_{i}>A_{i}, \\ \frac{t_{i}+A_{i}}{2 \Omega} R-\kappa_{i} \frac{t_{i}+A_{i}}{2} & 0<t_{i} \leq A_{i}, \\ 0 & t_{i}=0 .\end{cases}
$$

where $t_{i}$ is the strategized sensing time of user $i$, and $\Omega$ is the sum of expected sensing time of the users:

$$
\Omega=\sum_{i \in \mathcal{U}} \mathrm{E}\left(t_{i}+\varepsilon_{i}\right)=\sum_{j \in \mathcal{S}_{1}} \frac{t_{j}+A_{j}}{2}+\sum_{j \in \mathcal{S}_{2}} t_{j} .
$$

Here $\mathcal{S}_{1}, \mathcal{S}_{2}$ are the sets of the users such that $\forall i \in \mathcal{S}_{1}, 0<$ $t_{i} \leq A_{i}$ and $\forall i \in \mathcal{S}_{2}, t_{i}>A_{i} . \mathcal{S}=\mathcal{S}_{1} \cup \mathcal{S}_{2}$. Since the existence of uncertainty makes the crowdsourcing system a perturbed game, we have to consider expected utility rather than the pure utility. The utility of the platform is (4).

$$
\bar{u}_{0}=\lambda \log \left(1+\sum_{i \in \mathcal{U}} \mathrm{E}\left(t_{i}+\varepsilon_{i}\right)\right)-R
$$

where $\lambda>1$ is the revenue coefficient, and the log term shows the diminishing return of the platform on the sum of expected sensing time of users [7].

Trembling-Hand Perfect Equilibrium (THP) [22] is a refinement of Nash Equilibrium (NE). We give the related definitions as follows.

Definition 2. (Expected Best Response Strategy) Given the strategies of all the users except $i$, a strategy is user $i$ 's expected best response strategy, denoted by $\beta_{i}\left(t_{-i}\right)$, if it maximizes user $i$ 's utility $\mathrm{E}\left(\bar{u}_{i}\right)$.

Definition 3. (Trembling-Hand Perfect Equilibrium) The strategy profile $t^{e}=\left\{t_{i}^{e}\right\}_{i=1}^{+\infty}$ with uncertainty perturbation $\varepsilon=\left\{\varepsilon_{i}\right\}_{i=1}^{+\infty}$ is a Trembling-Hand Perfect Equilibrium of the perturbed game if there exists a sequence of totally mixed strategy profiles $\left\{t^{i}\right\}_{i=1}^{+\infty}$ s.t. $\lim _{i \rightarrow+\infty} t^{i}=t^{e}$ and $t_{i}=\beta_{i}\left(t_{-i}^{e}\right)$ for any user $i$. Here $\beta_{i}$ is user $i$ 's expected best response.

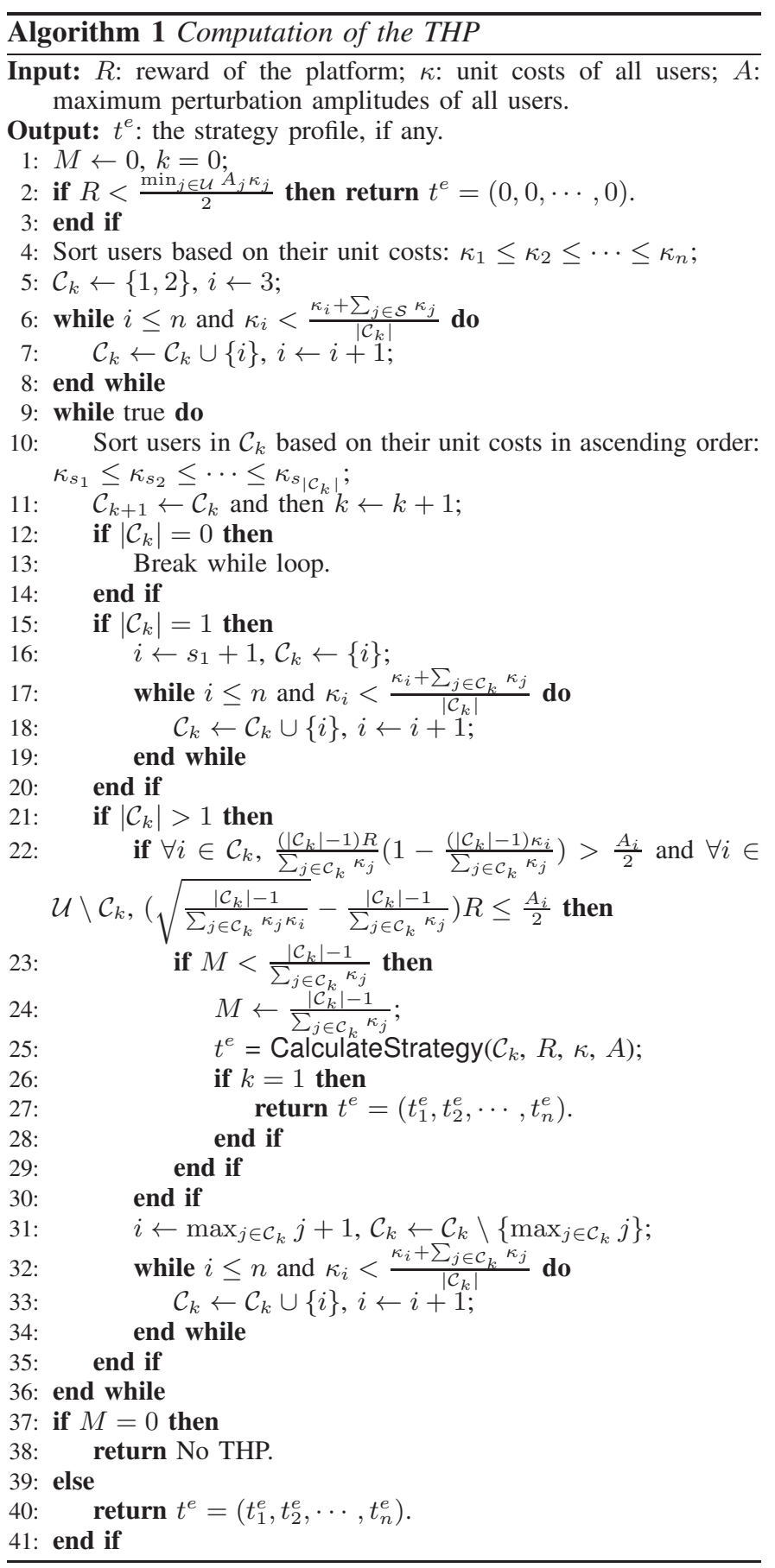

THP can guarantee that, even though the user cannot control its own sensing time accurately, it will gain nothing by choosing another strategy unilaterally in the long term. That means, the expected utility of any user is maximized given other users' strategies.

\section{Determining User Sensing Time With UNCERTAINTY PERTURBATION}

The classical game model with Nash Equilibrium has significant limitations when considering sensing time uncertainty in the crowdsensing game. To address this problem, we model the 


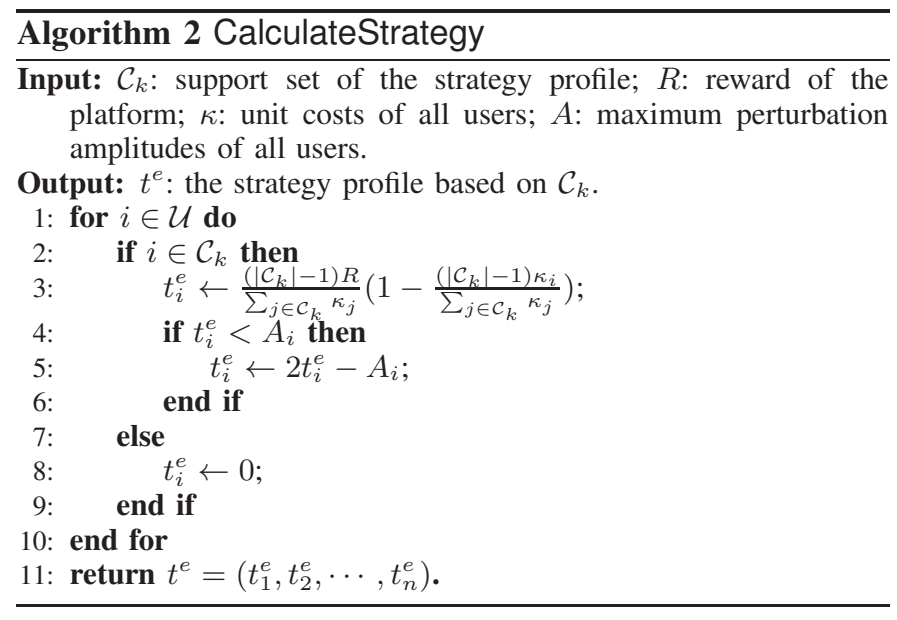

crowdsourcing system as a perturbed Stackelberg game with the solution concept Trembling-Hand Perfect Equilibrium as described in Section II-B. In this section, we will show how to find a THP strategy profile that optimizes the total sensing time of the perturbed game. Theorem 1 shows the sufficient condition to obtain THP in the game. Lemma 1 presents a trivial THP, in which no sensing happens. Theorem 2 shows how to calculate the non-trivial THP without the knowledge of other user's strategies. Based on our theoretic results, we propose Algorithm 1 to determine the user strategy profile that can achieve the maximum total sensing time.

\section{A. Trembling-Hand Perfect Equilibrium for Crowdsourcing Game with Uncertainty Perturbation}

The Trembling-Hand Perfect Equilibrium is a proper solution to adapt to the perturbations introduced by the uncertainty. Theorem 1 reveals that a strategy profile $\bar{t}$ s.t. $\forall i \in \mathcal{U}$, $\bar{t}_{i}=\beta_{i}\left(\bar{t}_{-i}\right)$ as defined in Definition 2 suffices that $\bar{t}$ is THP.

Theorem 1. For a strategy profile $\bar{t}$ of the perturbed game, if $\bar{t}_{i}=\beta_{i}\left(\bar{t}_{-i}\right)$ for any user $i, \bar{t}$ is THP.

We first discuss a trivial case of THP, in which no user will offer sensing service as stated in Lemma 1. Since there is no sensing work for trivial THP at all, we should avoid this case in our incentive mechanism design by letting the platform choose a proper reward. In Lemma $1, \mathcal{S}$ denotes the set of users with positive sensing time.

Lemma 1. 1) If $R<\frac{\min _{j \in \mathcal{U}} A_{j} \kappa_{j}}{2}$, there is a unique trivial $T H P t^{e}$ s.t. $\forall i \in \mathcal{U}, t_{i}^{e}=0$. 2) For non-trivial THPs, $|\mathcal{S}|>1$.

In order to find the non-trivial THP, based on Theorem 1 , we convert the equilibrium problem to the calculation of each user's expected best response strategy based on other users' strategies. By letting the equilibrium be the best strategy profile, we finish the calculation.

Theorem 2 shows how to determine a THP profile without knowledge of each user's strategy. Equation 5 gives the sum of the user strategies under THP. Equation 6 gives the user strategies only with costs $\kappa$, reward $R$ and perturbations $A$ as parameters.
Theorem 2. Let $t_{i}^{e}$ be non-trivial THP $(|\mathcal{S}| \geq 2)$ of the perturbed game. Then the following equations hold:

$$
\begin{array}{r}
\Omega=\sum_{j \in \mathcal{S}_{1}} \frac{t_{j}^{e}+A_{j}}{2}+\sum_{j \in \mathcal{S}_{2}} t_{j}^{e}=\frac{|\mathcal{S}|-1}{\sum_{j \in \mathcal{S}} \kappa_{j}} R, \\
t_{i}^{e}= \begin{cases}\frac{2(|\mathcal{S}|-1) R}{\sum_{j \in \mathcal{S}} \kappa_{j}}\left(1-\frac{|\mathcal{S}|-1}{\sum_{j \in \mathcal{S}} \kappa_{j}} \kappa_{i}\right)-A_{i} & i \in \mathcal{S}_{1}, \\
\frac{(|\mathcal{S}|-1) R}{\sum_{j \in \mathcal{S}} \kappa_{j}}\left(1-\frac{|\mathcal{S}|-1}{\sum_{j \in \mathcal{S}} \kappa_{j}} \kappa_{i}\right) & i \in \mathcal{S}_{2}, \\
0 & i \notin \mathcal{S} .\end{cases}
\end{array}
$$

And $\frac{(|\mathcal{S}|-1) R}{\sum_{j \in \mathcal{S}} \kappa_{j}}\left(1-\frac{|\mathcal{S}|-1}{\sum_{j \in \mathcal{S}} \kappa_{j}} \kappa_{i}\right)>\frac{A_{i}}{2}$ for any user $i \in \mathcal{S}$, and $\left(\sqrt{\frac{|\mathcal{S}|-1}{\sum_{j \in \mathcal{C}_{1}} \kappa_{j} \kappa_{i}}}-\frac{|\mathcal{S}|-1}{\sum_{j \in \mathcal{C}_{1}} \kappa_{j}}\right) R \leq \frac{A_{i}}{2}$ for any user $i \in \mathcal{U} \backslash \mathcal{S}$.

\section{B. Determining the Optimized THP}

We propose Algorithm 1 to find the THP that can achieve the maximum sum of expected sensing time $\Omega$. In fact, if we can achieve the maximized $\Omega$, we can also achieve the maximized platform utility $\bar{u}_{0}$. This is because based on (3) and (4), we have $\bar{u}_{0}=\lambda \log (1+\Omega)-R$ and it is easy to see $\bar{u}_{0}$ strictly increases as $\Omega$ increases. The main idea of this algorithm is to check each THP and find out the optimized solution. Each THP has a subset of users whose strategies (sensing time) are positive. Such user subset is called the support set of the equilibrium. Within the outer loop starting at line 9, Algorithm 1 keeps checking the members of the support set in order to find THPs. If our algorithm fails to find such a correct support set, the perturbed game actually does not have any THP. [18] shows that provided the proper reward $R$, the complexity of Algorithm 1 can be $O(n \log n)$ with the number of users. Solid theoretic analysis verifies that Algorithm 1 can efficiently find the THP to optimize the total sensing time given the reward $R$.

Theorem 3 shows the correctness of Algorithm 1.

Theorem 3. We assume there exists a THP of the game. Then we have:

1) The strategy profile $t^{e}=\left(t_{1}^{e}, t_{2}^{e}, \cdots, t_{n}^{e}\right)$ computed by Algorithm 1 is a THP of the game.

2) The strategy profile $t^{e}$ computed by Algorithm 1 maximizes system-wide total sensing time $\Omega$.

\section{Discussions on Algorithm 1}

Distributedness Algorithm 1 is highly distributed. That is, every user can compute Algorithm 1 independently. There is no required central coordination. However, since there are multiple THPs, the users may have inconsistence when choosing THP. To address this issue, instead of centralized algorithms, we use indirect control, for example, a proper reward to let the users agree on the optimized THP. This practice does a favor for the platform and makes the systemwide equilibrium unique, and Algorithm 1 can be computed efficiently. If any of the users unilaterally choose another suboptimized THP, they will take their own risk of utility loss.

Complexity If the game only has the trivial THP, Algorithm 1 exits at line 2 and it takes $O(n)$ time to locate the minimized 
$A_{i} \kappa_{i}$. If $\mathcal{C}_{1}$ is the support set of THP, Algorithm 1 returns at line 27. The time complexity is $O(n \log n)$ due to the sorting at line 10. In [18], we will show that a proper reward $R$ can guarantee such scenario.

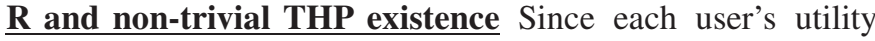
function is not continuous at the point $t_{i}=0$, our perturbed game is a discontinuous game. Thus our game does not have THP for some certain $R$ according to [23]. Algorithm 1 will return no THP if we cannot find the support set of THP. If there is no THP, the platform can increase the reward $R$ to a certain value in order to satisfy the non-trivial THP conditions. This is because for any such game, there exists a positive $R_{0}$ that for any $R>R_{0}$, there exists a non-trivial THP profile.

Theorem 4. For any game with more than 2 users, there exists a positive reward $R_{0}$ that for any reward $R>R_{0}$, there exists a non-trivial THP strategy profile $t^{e}$.

Sufficiently large reward can guarantee a non-trivial THP. However, too much reward will cause deficit to the platform. In fact [18], the platform can choose a proper $R$ before initiating the sensing work to ensure there exists at least one THP profile. We will elaborate it later.

\section{Simulation}

In order to evaluate the performance of our system, we implemented the incentive mechanism for the crowdsourcing game with uncertainty perturbation. We have the following performance metrics: 1) running time, 2) average user utility, 3) platform utility and 4) the number of participating users.

\section{A. Simulation Setup}

For the crowdsourcing game with uncertainty perturbation, we assume the cost unit $\kappa$ of each user was distributed uniformly over $\left[1, \kappa_{\max }\right]$, and $\left|A_{i}\right|$ of any user $i$ was distributed uniformly over $\left[0, A_{\max }\right]$. There are four variables: a) number of users $n$ (from 100 to 1000 with the increment of 100), b) the revenue coefficient $\lambda$ (from 10 to 100 with the increment of 10), c) $\kappa_{\max }$ (from 1 to 10 with the increment of 1) and d) $A_{\max }$ (from 0 to 1 with the increment of 0.1). If we need to fix some variables, the default values are: $n=1000, \lambda=50$, $\kappa_{\max }=5$ and $A_{\max }=0.5$. The simulations were run on a Linux workstation (2.0 GHz CPU and $32 \mathrm{~GB}$ memory). Each result is averaged over 1000 instances.

\section{B. Simulation of the Crowdsourcing Incentive Mechanism with Uncertainty Perturbation}

- Running Time: The running time of the incentive mechanism includes strategizing the reward and the THP profile. Fig. 2 shows the impact of the number of users on running time. The running time increases linearly with the number of users, and it is less than $5.00 \mathrm{~ms}$ for 1000 users.

- Platform Utility $\bar{u}_{0}$ : We analyze the impact of $A_{\max }$, $K_{\max }$ on $\bar{u}_{0}$. Fig. 3 shows: 1$)$ If $\kappa_{\max }$ is fixed, $\bar{u}_{0}$ reduces greatly when the uncertainty perturbations get more diverse; 2) Without sensing uncertainty perturbations, $\bar{u}_{0}$ diminishes when $\kappa_{\max }$ increases, as [7] demonstrated.
- Average User Utility: We analyze the impact of $A_{\max }$, $K_{\max }$ on average user utility $\bar{u}=\frac{1}{n} \sum_{i \in \mathcal{U}} \bar{u}_{i}$. Fig. 4 shows $\bar{u}$ increases when the cost units and uncertainty characteristics get more diverse. In this case, the platform will release more reward [18]. Thus $\bar{u}$ will increase as well.

\section{RELATED WORKS}

For the crowdsourcing with each user determines its own sensing time, Yang et. al [7] proposed the first incentive mechanism using Nash Equilibria to maximize the users and the platform's utilities. As Nash Equilibrium has strong assumptions on the complete information, it derives several refinements, such as Bayesian Perfect Equilibrium [24] and Trembling-Hand Perfect Equilibrium [25][26][27][28]. Azar et al. [29] designed auction-based incentive mechanism by modeling the crowdsourcing as a Bayesian game. To the best of our knowledge, there is not yet any incentive mechanism using Trembling-Hand Perfect Equilibrium for crowdsourcing. Besides, many other existing works focused on auction-based and strategy-proof incentive mechanism of crowdsourcing [30] [31] [32] [33] [34] [35]. The objective of auction-based incentive mechanism is to stimulate the participating users at the finest granularity [30]. Afuah et al. [31] studied local searchbased auction for crowdsourcing, giving local search algorithm to solve crowdsourcing problems efficiently. Lev et al. [32] researched the case of bidder collusion in all-pay auction and found several positive effects of collusion on crowdsourcing. On the other hand, researchers used strategy-proof incentive mechanism to avoid cheating in bidding, i.e. false-reporting sensing costs [36]. Huang et al. [33] studied strategy-proof and privacy preserving spectrum auction mechanism. Babaioff et al. [34] modeled crowdsourcing as multiple buyers and the single seller with limited supply, and proposed strategy-proof mechanism to defend against buyers' collusion and privacy leakage. Koutsopoulos [35] gave a detailed theoretic treatment on truthful crowdsensing incentive mechanisms. Clearly the researchers are considering more and more real-world issues of crowdsourcing, such as truthfulness and privacy preservation.

\section{CONCLUSION}

In this paper, we have designed incentive mechanisms for crowdsourcing with sensing time uncertainty. Our contributions have filled the void of the research of crowdsourcing with uncertainty. We have introduced the perturbed game model and used Trembling-Hand Perfect Equilibria to maximize each user's utility. We have proposed the algorithm to calculate THP of the perturbed game, if it exists. Extensive experiments have rigorously verified our incentive mechanism is incentivecompatible, optimal and efficient.

\section{REFERENCES}

[1] N. D. Lane, E. Miluzzo, H. Lu, D. Peebles, T. Choudhury, and A. T. Campbell, "A survey of mobile phone sensing," Communications Magazine, IEEE, vol. 48, no. 9, pp. 140-150, 2010.

[2] R. K. Ganti, K. Raghu, F. Ye, and H. Lei, "Mobile crowdsensing: Current state and future challenges," Communications Magazine, IEEE, vol. 49, no. 11, pp. 32-39, 2011. 


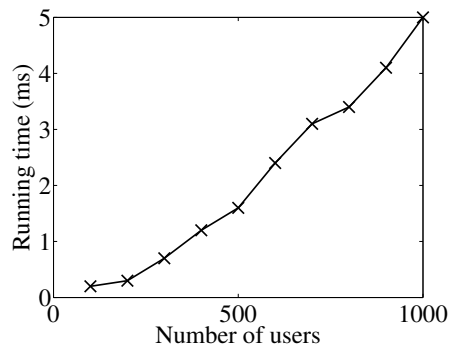

Fig. 2. Impact of the number of users on running time

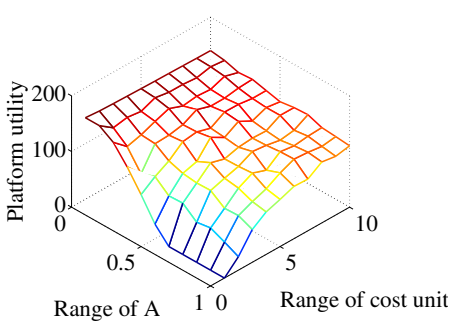

Fig. 3. Impact of $A_{\max }$ and $\kappa_{\max }$ on platform utility

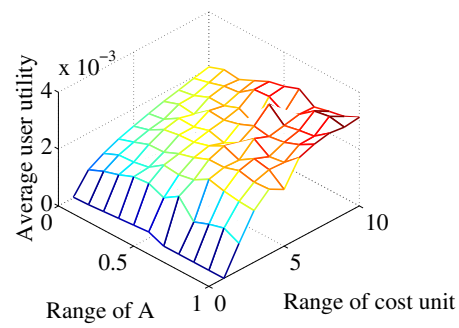

Fig. 4. Impact of $A_{\max }$ and $\kappa_{\max }$ on average user utility
[3] G. Chatzimilioudis, A. Konstantinidis, C. Laoudias, and D. ZeinalipourYazti, "Crowdsourcing with smartphones," Internet Computing, IEEE, vol. 16, no. 5, pp. 36-44, 2012.

[4] M. Mun, S. Reddy, K. Shilton, N. Yau, J. Burke, D. Estrin, M. Hansen, E. Howard, R. West, and P. Boda, "Peir: the personal environmental impact report, as a platform for participatory sensing systems research," in Proc. ACM/USENIX Int. Conf. Mobile Systems, Applications, and Services (MobiSys) Krakow, 2009.

[5] K. K. Rachuri, M. Musolesi, C. Mascolo, P. J. Rentfrow, C. Longworth, and A. Aucinas, "Emotionsense: a mobile phones based adaptive platform for experimental social psychology research," in Proceedings of the 12th ACM international conference on Ubiquitous computing, ser. Ubicomp '10. New York, NY, USA: ACM, 2010, pp. 281-290.

[6] B. Kim, J.-Y. Ha, S. Lee, S. Kang, Y. Lee, Y. Rhee, L. Nachman, and J. Song, "Adnext: a visit-pattern-aware mobile advertising system for urban commercial complexes," in Proceedings of the 12th Workshop on Mobile Computing Systems and Applications, ser. HotMobile '11. New York, NY, USA: ACM, 2011, pp. 7-12.

[7] D. Yang, G. Xue, X. Fang, and J. Tang, "Crowdsourcing to smartphones: incentive mechanism design for mobile phone sensing," in Proceedings of the 18th annual international conference on Mobile computing and networking. ACM, 2012, pp. 173-184.

[8] X. Sheng, X. Xiao, J. Tang, and G. Xue, "Sensing as a service: A cloud computing system for mobile phone sensing," in Sensors, 2012 IEEE, October 2012

[9] M.-R. Ra, B. Liu, T. F. La Porta, and R. Govindan, "Medusa: A programming framework for crowd-sensing applications," in Proceedings of the 10th international conference on Mobile systems, applications, and services. ACM, 2012, pp. 337-350.

[10] Y. Chon, N. D. Lane, F. Li, H. Cha, and F. Zhao, "Automatically characterizing places with opportunistic crowdsensing using smartphones," in Proceedings of the 2012 ACM Conference on Ubiquitous Computing. ACM, 2012, pp. 481-490.

[11] Y. Chon, N. D. Lane, Y. Kim, F. Zhao, and H. Cha, "Understanding the coverage and scalability of place-centric crowdsensing," in Proceedings of the 2013 ACM international joint conference on Pervasive and ubiquitous computing. ACM, 2013, pp. 3-12.

[12] D. J. Hand, "Principles of data mining," Drug safety, vol. 30, no. 7, pp. 621-622, 2007

[13] M.-S. Chen, J. Han, and P. S. Yu, "Data mining: an overview from a database perspective," Knowledge and data Engineering, IEEE Transactions on, vol. 8, no. 6, pp. 866-883, 1996.

[14] J. C. Prather, D. F. Lobach, L. K. Goodwin, J. W. Hales, M. L. Hage, and W. E. Hammond, "Medical data mining: knowledge discovery in a clinical data warehouse." in Proceedings of the AMIA Annual Fall Symposium. American Medical Informatics Association, 1997, p. 101.

[15] U. Fayyad, G. Piatetsky-Shapiro, and P. Smyth, "From data mining to knowledge discovery in databases," AI magazine, vol. 17, no. 3, p. 37, 1996.

[16] A. Charland and B. Leroux, "Mobile application development: web vs. native," Communications of the ACM, vol. 54, no. 5, pp. 49-53, 2011.

[17] Y. Wang, J. Lin, M. Annavaram, Q. A. Jacobson, J. Hong, B. Krishnamachari, and N. Sadeh, "A framework of energy efficient mobile sensing for automatic user state recognition," in Proceedings of the 7th international conference on Mobile systems, applications, and services. ACM, 2009, pp. 179-192.

[18] S. Ji, T. Chen, and F. Wu, "Crowdsourcing with trembles: Incentive mechanisms for error-prone mobile phone sensing," in Technical Report, Oklahoma State University., July 2013. [Online]. Available: http://goo.gl/F4P0PK

[19] T. Basar, G. J. Olsder, G. J. Clsder, T. Basar, and T. Baser, Dynamic noncooperative game theory. London: Academic press, 1995, vol. 200.

[20] J. Burkardt, "The truncated normal distribution," Department of Scientific Computing Website, Florida State University, 2014.

[21] P. Damien and S. G. Walker, "Sampling truncated normal, beta, and gamma densities," Journal of Computational and Graphical Statistics, vol. 10, no. 2, 2001.

[22] L. K. Simon and M. B. Stinchcombe, "Equilibrium refinement for infinite normal-form games," Econometrica: Journal of the Econometric Society, pp. 1421-1443, 1995.

[23] O. Carbonell-Nicolau, "The existence of perfect equilibrium in discontinuous games," Games, vol. 2, no. 3, pp. 235-256, 2011.

[24] J. C. Harsanyi, "Games with incomplete information played by bayesian players, i-iii," Management Science, vol. 50, no. 2, pp. 1804-1817, 2004.

[25] R. Selten, "A reexamination of the perfectness concept for equilibrium points in extensive games," International journal of game theory, vol. 4, no. 1, pp. 25-55, 1975.

[26] E. Van Damme and S. Hurkens, "Games with imperfectly observable commitment," Games and Economic Behavior, vol. 21, no. 1, pp. 282 308, 1997.

[27] D. H. Wolpert, "Trembling hand perfection for mixed quantal/best response equilibria," International Journal of Game Theory, vol. 38, no. 4, pp. 539-551, 2009.

[28] P. C. Mittal, S. K. Yadav, and K. Sharma, "Trembling hand perfection in repeated two stackelberg leader and follower," Dronacharya Research Journal, vol. 3, no. 2, 2011.

[29] P. Azar, J. Chen, and S. Micali, "Crowdsourced bayesian auctions," in Proceedings of the 3rd Innovations in Theoretical Computer Science Conference, January 2012, pp. 236-248.

[30] B. Satzger, H. Psaier, D. Schall, and S. Dustdar, "Stimulating skill evolution in market-based crowdsourcing," Business Process Management, vol. 6896, pp. 66-82, 2011.

[31] A. Afuah and C. L. Tucci, "Crowdsourcing as a solution to distant search," Academy of Management Review, vol. 37, no. 3, pp. 355-375, 2012.

[32] O. Lev, M. Polukarov, Y. Bachrach, and J. S. Rosenschein, "Mergers and collusion in all-pay auctions and crowdsourcing contests," in Proceedings of the 2013 international conference on Autonomous agents and multi-agent systems (AAMAS '13), May 2013, pp. 675-682.

[33] Q. Huang, Y. Tao, and F. Wu, "Spring: A strategy-proof and privacy preserving spectrum auction mechanism," in IEEE International Conference on Computer Communications (IEEE INFOCOM 2013), April 2013, pp. 14-19.

[34] M. Babaioff, S. Dughmi, R. Kleinberg, and A. Slivkins, "Dynamic pricing with limited supply," in Proceedings of the 13th ACM Conference on Electronic Commerce, June 2012, pp. 74-91.

[35] I. Koutsopoulos, "Optimal incentive-driven design of participatory sensing systems," in INFOCOM, 2013 Proceedings IEEE. IEEE, 2013, pp. $1402-1410$

[36] Z. Feng, Y. Zhu, and L. M. Ni, "imac: Strategy-proof incentive mechanism for mobile crowdsourcing," Wireless Algorithms, Systems, and Applications, vol. 7992, pp. 337-350, 2013. 